\title{
Billions of Human Brains Immersed Within a Shared Geomagnetic Field: Quantitative Solutions and Implications for Future Adaptations
}

\author{
Michael A. Persinger* \\ Department of Biology and Behavioural Neuroscience and Biomolecular Sciences Programs, Laurentian University, \\ Sudbury, Ontario, Canada P3E 2C6
}

\begin{abstract}
The implications for adaptation when billions of human cerebrums are considered weak conductors immersed within the same medium, the geomagnetic field, are examined. Quantitative solutions indicated that the intensity of the "transcerebral" field produced from all human brains within the geomagnetic field is the same order of magnitude as the values associated with cognitive processes and altered expressions of proteins within the individual brain. This convergence could meet one of the criteria for a holographic-like phenomenon. The transition from 6 to 8 billion brains would be associated with shared energies within individual cerebral space whose frequencies increase across the visible electromagnetic wavelength from infrared to ultraviolet. Magnetic diffusivity indicates all brains could be influenced within about 10 minutes. Implications for induced ubiquitous genetic changes, shared modifications in protein sequences associated with memory during dream sleep, and limitations upon the proliferation of the species are discussed.
\end{abstract}

Keywords: Geomagnetic field, cerebral fields, photon emission, genetic medication, magnetic diffusivity, population control.

\section{INTRODUCTION}

The human species has been and continues to be immersed within the earth's static magnetic field. Its general intensity averages around $50,000 \mathrm{nT}(0.5$ gauss $)$ with a discrepancy of approximately a factor of 2 to 3 between the equatorial and polar regions [1]. Living areas above large aggregates of near-surface magnetized minerals, such as the Kursk anomaly in the Ukraine, are about 4 times the average intensity. Geomagnetic fluctuations, primarily from alterations in solar activity mediated by changes in the velocity and density of particles in the interplanetary magnetic field (the "solar wind"), are one-thousandth to one hundredth of this steady-state intensity. The durations of these fluctuations are in the order of minutes with median ranges of intensity in the order of 50 to $1000 \mathrm{nT}$.

This ratio is comparable to the differences in magnitude between the steady-state potential differences $(\sim 10$ to 20 $\mathrm{mV}$ ) between the rostral and caudal distances of the human cerebrum and the primary time-varying, extremely low frequency fluctuations measured over the cerebral cortices. The majority of the power within this extremely low frequency band is between 1 and $40 \mathrm{~Hz}$ with peak to peak magnitudes between 10 and $200 \mu \mathrm{V}$. Conventional measurements by modern quantitative electroencephalographic (QEEG) technology usually range from 1-10 $\mu \mathrm{V} \cdot \mathrm{Hz}^{-1}[2]$.

Increases in indices of global geomagnetic activity, particularly when disturbances exceed about $25 \mathrm{nT}$, have

*Address correspondence to this author at the Department of Biology and Behavioural Neuroscience and Biomolecular Sciences Programs, Laurentian University, Sudbury, Ontario, Canada P3E 2C6; Tel: 01-705-675-4824;

Fax: 01-705-671-3844; E-mail: mpersinger@laurentian.ca been associated with increased incidence of epileptic seizures, unusual nocturnal experiences, and subsequent alterations in behaviors within vulnerable populations [3]. Experimental simulations of the intensity and temporal shapes of geomagnetic activity in the laboratory produce comparable changes [4]. Recently Mulligan et al. [5] in Canada replicated the results of Babayev and Allahveriyeva [6] in Azerbaijan that changes in cerebral power as measured by QEEG occurred during increases in geomagnetic activity above 25 to $30 \mathrm{nT}$. The most consistent, moderate strength correlations were evident for theta $(4 \mathrm{to} 7 \mathrm{~Hz})$ and gamma (35 to $45 \mathrm{~Hz}$ ) frequency bands over the right frontal lobe which displays a fundamental role in the reconstruction of autobiographical memory [7]. Mulligan et al. [5] calculated that the energy densities within the cerebral volume from the global increase in geomagnetic activity were the same order of magnitude as those associated with the numbers of cortical neurons necessary to produce the changes in QEEG measurements.

However the cerebral changes associated with geomagnetic activity are transient. Most of the biological time the human species is continuously exposed to the more or less "steady-state" or static component of the earth's surface magnetic field. This simultaneous immersion of about 6 to 7 billion human brains, that are effectively very similar semiconducting microstructures within this magnetic field, may be sufficient to produce a secondary field that may have biological implications for survival and adaptation. This secondary field could display emergent properties with qualitatively different characteristics. In previous approaches [8] metaphoric references to the secondary field generated by exposing large numbers of conductors (such as functionally adjacent copper wires) to an applied magnetic field have been employed to describe this condition. Within the present 
paper quantitative solutions for this model are offered that may be relevant to testing the validity of this approach. If the solutions are even partially applicable, the implications for the mechanisms by which our species could adapt would require a re-evaluation of our most fundamental assumptions of evolutionary and ecological principles.

\section{THE MODEL AND QUANTITATIVE SOLTUIONS}

The assumptions for the current model are derived from established physical parameters. The orders of magnitude for these geophysical and biological values have been more or less the same for decades. The coefficients have changed as a function of greater sample sizes or more precise instrumentation. I assume a normal distribution of the dispersion of individual values around the central tendency. Although this may appear to introduce imprecision, the consistency of the orders of magnitude for the parameters in general dominate the stability of values. However where relevant, ranges of the solutions, are given.

The adult volume of the human brain is about $10^{-3} \mathrm{~m}^{3}$. The change in volume since mean weights were initially published in 1880 [9] have ranged from $1.296 \mathrm{~kg}$ to $1.445 \mathrm{~kg}$ for males and $1.170 \mathrm{~kg}$ to $1.307 \mathrm{~kg}$ for females. The mean volume of $0.1 \mathrm{~kg}$ of fresh brain tissue is $\sim 9.1 \cdot 10^{-5} \mathrm{~m}^{3}$. For this model I assume that all living cerebrums are immersed within the geomagnetic field that is coupled to a vertical current density associated with ionospheric-ground currents. Both the static geomagnetic field and these electric currents are considered because they are intricately connected [10].

In this paper an average of about $10^{-13} \mathrm{~A} \cdot \mathrm{m}^{-2}$ is assumed to be an intermediate between the 2 to $3 \cdot 10^{-12} \mathrm{~A} \cdot \mathrm{m}^{-2}$ over land [11] and $\sim 10^{-14} \mathrm{~A} \cdot \mathrm{m}^{-2}$ over the oceans [12] which is remarkably similar to the values recorded by Koenig et all in his comprehensive text [13]. More precisely their measurements clearly indicated 0.5 to $5 \cdot 10^{-14} \mathrm{~A} \cdot \mathrm{m}^{-2}$, or, $10^{-13} \mathrm{~A} \cdot \mathrm{m}^{-2}$ which is the same as those reported by more recent estimations [14]. From traditional calculations [15] of magnetic field strength ( $\mathrm{B}$, or as units: $\mathrm{kg} \cdot \mathrm{A}^{-1} \cdot \mathrm{s}^{-2}$ ) from current within conductors distributed as a large current sheet, the relation is:

$\mathrm{B}=\mu \cdot \mathrm{i} \cdot \mathrm{d}$

where $\mu=$ magnetic permeability $\left(1.26 .10^{-6} \mathrm{~kg} \cdot \mathrm{m} \cdot \mathrm{s}^{-2} \cdot \mathrm{A}^{-2}\right)$, $\mathrm{i}$ is current density $\left(10^{-13} \mathrm{~A} \cdot \mathrm{m}^{-2}\right)$, and $\mathrm{d}$ is the length $(\mathrm{m})$ within which the current is applied.

The uncertain component here is the sum of length, d. Here I assume that length of all cerebrums is functionally connected within the geomagnetic field. According to Blinkov and Glezer [9] the actual range in the three spatial dimensions of the cerebrum for their samples (which is very similar to what I have measured over the last 40 years) were 11.8 to $16.3 \mathrm{~cm}(\mathrm{M}=14 \mathrm{~cm})$ for width, 14.1 to $18.3 \mathrm{~cm}$ $(\mathrm{M}=16.7 \mathrm{~cm})$ for length and 7.3 to $10.4 \mathrm{~cm}(\mathrm{M}=9.3 \mathrm{~cm})$ for height. Here I am assuming a functional average length of $\sim 11 \mathrm{~cm}$ for two reasons. First, it is a minimum value that would satisfy the adult brain and include the currently developing brains within the species. Second, when this value is assumed for calculating the functional volume of the cerebrum, exact solutions in Minkowskian (4-dimensional) space [16] are congruent with universal parameters relevant to biophysical and quantum functions within brain space. This is relevant to the emerging field of Quantum Biology.

Assuming 6 billion brains each with an average length of $1.1 \cdot 10^{-1} \mathrm{~m}$, the total length is $6.6 \cdot 10^{8} \mathrm{~m}$. The strength of the induced magnetic field would be $\sim 83 \mathrm{pT}$. Assuming 7 billion brains, to appreciate the effect of population numbers, the induced magnetic field would be $\sim 97 \mathrm{pT}$. Between 150 and 200 years ago when the human population was about 1 billion, the value would be $\sim 14$ pT. This "induced transcerebral field" would be the consequence of the billions of conductors sharing the same geomagnetic medium. For comparison the averaged magnetic field of the action potential $1 \mathrm{~mm}$ from an isolated sciatic nerve of a frog is between 60 and $125 \mathrm{pT}$ [17]. This distance $(1 \mathrm{~mm})$ is very relevant within cerebral cortical space. It is within the range of the width of cortical columns as well as the hundreds of foci of intrinsic curvatures primarily concentrated along the crests of gyri and the fundi of sulci that were recently revealed by amplified quantitative functional Magnetic Resonance Imaging [18].

This predicted order of magnitude associated with this hypothetical transcerebral magnetic field induced between 6 and 7 billion human brains on earth is similar to the magnetic field strength associated with cerebral processes within the individual human brain [19]. When the magnitude of the whole field equals or approaches the magnitude of the individual unit $(n)$, that is $\sum n=n$, the conditions for a hologram emerge [20]. Metaphorically this allows for the information of the whole field to be represented within a unit and the information within the unit to be represented within the whole field [21].

The potential energy that could be maintained within the geomagnetic field is significant. The product of its dipole moment $\left(8 \cdot 10^{22} \mathrm{~A} \cdot \mathrm{m}^{2}\right)$ and average intensity $\left(5 \cdot 10^{-5} \mathrm{~T}\right.$ or $\left.\mathrm{kg} \cdot \mathrm{A}^{-1} \cdot \mathrm{s}^{-2}\right)$ is about $4 \cdot 10^{18} \mathrm{~J}$. With $\sim 10^{-20} \mathrm{~J}$ per action potential associated with each unit charge [22], an average discharge of $\sim 10 \mathrm{~Hz}$ (range of alpha rhythms) and $4 \cdot 10^{10}$ neurons in the cerebral cortices, the life-time $(\sim 2 \mathrm{Gsec})$ electromagnetic energy from the information associated with this activity from each human cerebrum would be about $1 \mathrm{~J}$. Assuming 50 billion human brains in recent history, the total energy associated with cerebral activity (and the subsequent alterations in synaptic activity associated with memory) would have involved $\sim 10^{11} \mathrm{~J}$. Even if there were diminishment in the magnitude of the dipole moment by factors of $100 \mathrm{~s}$, there would still be sufficient potential to represent all of this information.

This means the earth's magnetic field has sufficient capacity to represent or "store" the information within the energy that has been associated with the action potentials that have been generated by every human brain that has existed on the planet [23]. If, as the Landauer Limit predicts, a change in one bit of information or the convergence of two operations, is associated with a loss or gain of $\ln 2 \cdot \mathrm{kT}$, where $\mathrm{k}$ is the Boltzmann constant $\left(1.38 \cdot 10^{-23} \mathrm{~J} \cdot \mathrm{K}^{-1}\right)$ and $\mathrm{T}$ is the temperature $\left(310^{\circ} \mathrm{K}\right)$, then extremely large numbers of quanta of energy could be represented as information within this matrix. Such convergence does not prove that such representation exists. However a quantized medium might exist that would allow the condition for connectivity at several levels of discourse [24]. 


\section{LATENCY FOR CONNECTION BETWEEN BRAINS}

The surface area $\left(\sim 5 \cdot 10^{14} \mathrm{~m}^{2}\right)$ of the planet is much larger with respect to the cross sectional area of each brain or the summed area of the approximately 7 billion human brains. The critical variable for the unit brains to behave as a field or as a whole and to exhibit holographic-like properties is the latency for "connection". In other words what would the latency in real time between a critical change in one cerebrum that would potentially diffuse through all of the other brains within this induced transcerebral field? This process can be inferred by magnetic diffusivity $\left(\mathrm{m}^{2} \cdot \mathrm{s}^{-1}\right)$ which is defined as $\sigma \cdot \mu^{-1}$ where $\sigma$ is conductivity [25].

For physiological saline (within each brain) $\sigma=2.1 \mathrm{~S} \cdot \mathrm{m}^{-1}$, which results in a magnetic diffusivity of $1.3 \cdot 10^{6} \mathrm{~m}^{2} \cdot \mathrm{s}^{-1}$. The surface area of each cerebrum is assumed to be $\sim \pi \cdot 10^{-2} \mathrm{~m}^{2}$ and hence the total surface areas for 6 and 7 billion brains would be $18.8 \cdot 10^{7} \mathrm{~m}^{2}$ and $22 \cdot 10^{7} \mathrm{~m}^{2}$, respectively. The time required for an integrated magnetic diffusivity for these areas would range between be $1.3 \cdot 10^{6} \mathrm{~m}^{2} \cdot \mathrm{s}^{-1}$ divided by $18.8 \cdot 10^{7}$ $\mathrm{m}^{2}$ or $22 \cdot 10^{7} \mathrm{~m}^{2}$, or between $143 \mathrm{~s}(2.4 \mathrm{~min})$ and $168 \mathrm{~s}(2.8$ $\mathrm{min}$ ). If average tissue conductance, $\sigma=0.5 \mathrm{~S} / \mathrm{m}$, was considered instead the solutions would range between 482 and $564 \mathrm{~s} \mathrm{(} \sim 8$ to $9 \mathrm{~min})$. Even with this range of conductance values within cerebral tissue, the theoretical time for a critical change in one brain to encompass all other cerebra in the field would be similar to important biological processes such a single episode of dreaming or the latency for the formation of the spines on dendrites during the initial stages of memory consolidation. Interestingly this is also the duration for excess correlations ("entanglement") of flux densities to occur between fields of photons emitted from two, separate chemical reactions separated by non-local distances [26].

The range of these durations also overlaps with the band of periodicity, between $3 \mathrm{mHz}$ and $5 \mathrm{mHz}$, that defines the peak amplitude (about $5 \cdot 10^{-12} \mathrm{~m} \cdot \mathrm{s}^{-2}$ ) of the free oscillations between the solid earth and the atmosphere [27]. The effect is not restricted to these small changes in the approximately $\mathrm{pN}\left(10^{-12}\right.$ to $\left.10^{-11} \mathrm{~N}\right)$ that would be exerted on a $1.5 \mathrm{~kg}$ cerebral mass. The annual variations of the spectral power density of the amplitudes of these free oscillations of the earth are also correlated with the amplitudes of the spectral power densities of ultraweak photon emissions from the earth [28]. The potential existence of "fields of photons", with spectral power densities within the order of $10^{-13} \mathrm{~W} \cdot \mathrm{m}^{-2}$, the same order of magnitude as the energy from background cosmic rays at sea level [13], could suggest the presence of a conduit. That very small energies and one unit contained within tens of millions of units can determine the function of the whole has been shown for brain activity. Recently Li et al. [29] demonstrated experimentally that burst spiking of a single cortical neuron modified the global brain state.

It is relevant that the current decay within the electric field between the earth's surface and ionosphere can be obtained by dividing the permittivity of space $e_{0}=8.85 \cdot 10^{-12}$ $\mathrm{F} \cdot \mathrm{m}^{-1}$ by the average conductivity of $\sim 2 \cdot 10^{-14} \mathrm{~S} \cdot \mathrm{m}^{-1}$. The value is in the order of $7 \mathrm{~min}$. The role of the Schumann-type frequencies (fundamental around 7 to $8 \mathrm{~Hz}$ ) may be relevant as well [30]. This frequency is in large part determined by the fixed relationship between the velocity of light $\left(3 \cdot 10^{8}\right.$ $\left.\mathrm{m} \cdot \mathrm{s}^{-1}\right)$ and the earth's circumference $\left(\sim 4 \cdot 10^{7} \mathrm{~m}\right)$. The total magnetic flux of the earth's surface $\left(5.1 \cdot 10^{14} \mathrm{~m}^{2}\right)$ for an average global value of $5 \cdot 10^{-5} \mathrm{~T}$ is $2.6 \cdot 10^{10}$ Webers. The amperage for this field would be the dipole moment $\left(8 \cdot 10^{22}\right.$ $\mathrm{A} \cdot \mathrm{m}^{2}$ ) divided by the surface area, or $1.6 \cdot 10^{8} \mathrm{~A}$. Therefore the inductance, which is Weber per amp, would be $1.6 \cdot 10^{2}$ Henrys. With this value for inductance, a global capacitance of 2 Farads [31], and a frequency of 7-8 Hz, the solution would be $2 \mathrm{ks}$ or about $30 \mathrm{~min}$. These values are within the range required for consolidation of memory (the representation of experience) from the electrically labile stage to the patterns associated with the synthesis of proteins and the growth of dendritic spines at synapses [32].

\section{SECONDARY INTRACERERBAL MAGNETIC FIE- LDS AND PHOTON EMISSIONS}

The energy within a magnetic field for a given volume is traditionally [15] defined as:

$\mathrm{J}=\mathrm{B}^{2} \cdot(2 \mu)^{-1} \cdot \mathrm{m}^{3}$

Consequently the energy induced within the volume of each cerebrum by the hypothetically induced cerebral field produced by 6 billion human brains being immersed within the earth's magnetic field would be $\left[\left(8.3 \cdot 10^{-11} \mathrm{~T}\right)^{2} / 2.5 \cdot 10^{-6}\right.$ $\mathrm{N} / \mathrm{A}] \cdot 1.3 \cdot 10^{-3} \mathrm{~m}^{3}$, or $3.5 \cdot 10^{-19} \mathrm{~J}$. The frequency associated with this value is obtained by dividing by Planck's constant $\left(6.62 \cdot 10^{-34} \mathrm{~J} \cdot \mathrm{s}\right)$ which results in $0.54 \cdot 10^{15} \mathrm{~Hz}$. At $\mathrm{c}$, the velocity of light in a vacuum, the equivalent wavelength would be $555 \mathrm{~nm}$ or within the range of visible light (400 to $800 \mathrm{~nm})$.

That normal cerebral activity can generate photons within this band was predicted theoretically [33] and recently shown experimentally [34]. The photon emission power densities associated with certain types of cognitive activity, such as thinking about white light while sitting in darkness, are in the order of $10^{-11} \mathrm{~W} \cdot \mathrm{m}^{-2}$ or, when the cross-sectional cerebral space is accommodated, would be $10^{-13} \mathrm{~J} \cdot \mathrm{s}^{-1}$. This is equivalent to the energy associated with 10 million cortical neurons discharging once per sec [22]. Approximately 10 million cortical neurons are typically activated, as inferred from integrated fMRI voxels, during specific cognitive activities.

For comparison the earth's free oscillations [27], including that between the solid earth and atmosphere, display amplitudes of the order of $5 \cdot 10^{-12} \mathrm{~m} \cdot \mathrm{s}^{-2}$. When applied across the mass of a human cerebrum and its length, is also within the order of $10^{-13} \mathrm{~J}$. Most natural phenomena that share pathways or are intercorrelated involve functional components or processes that display remarkably similar increments of energy. The convergence would be consistent with the existence of a common conduit between gradations of global energy levels generated by the earth and the energy associated with human cerebral activity.

The sum of all energy from the quantum of $3.5 \cdot 10^{-19} \mathrm{~J}$ induced by the transcerebral magnetic field within six billion cerebrums would be $2.1 \cdot 10^{-9} \mathrm{~J}$. For comparison this is within a few percent of the estimated summed energy per second associated with each action potential $\left(2 \cdot 10^{-20} \mathrm{~J}\right)$ generated by the approximately 25 billion $\left(2.5 \cdot 10^{10}\right)$ neurons within a hemisphere each discharging within the 7-8 $\mathrm{Hz}$ range [35]. The latter is also the primary resonance frequency for the 
cerebrum given its inductance and capacitance [36] and the measured peak frequency within the cerebral volume [37].

The 7-8 Hz increment is the primary oscillation mode of the parahippocampal area through which the entire cerebral cortices can be accessed [38] and of the hippocampal formation [39] which is the primary locus for the electromagnetic and chemical processes that maintain the labile representations of experience until their equivalents as patterns of spines on dendrites emerge after about $20 \mathrm{~min}$ [32]. It is also the fundamental (Schumann) frequency associated with the earth's unique spherical resonator between the earth's surface and ionosphere [30]. Within this cavity, where conductivity is $\sim<10^{-10} \mathrm{~S} \cdot \mathrm{m}^{-1}$ the functional frequency would be within the range of cortical activity particularly during high energy particle precipitation due to solar proton events [40].

That energy within the visible wavelength could occur within each cerebrum because of the secondary magnetic field induced by "cerebral" field is significant. The stacking energies for nucleotides for synthesizing strands of RNA and DNA are about $10^{-20} \mathrm{~J}$. For human beings (as well as E. coli) the mutation rate is approximately 1 nucleotide per $10^{9}$ nucleotides for each DNA replication [41]. A single deletion or addition of a base pair that would have been added if the increment of energy had not been present would be sufficient to affect the subsequent type of protein synthesis as determined by the triplet pairs of nucleotides. The continuous background energy of $\sim 10^{-19} \mathrm{~J}$ of light indicates that as many as $10,10^{-20} \mathrm{~J}$ equivalents [23] could occur within each cerebrum per second. Over intervals of days to weeks, the potential alteration or even homogeneity of specific types of protein patterns within all cerebral volumes distributed over the surface of the earth would be expected to increase.

The biochemical consequence is not trivial. Some neuropeptides and proteins display functional concentrations within the femtomole $\left(10^{-15} \mathrm{M}\right)$ to attomole $\left(10^{-18} \mathrm{M}\right)$ range, assuming minimal degradation. Within a single day of $8.64 \cdot 10^{4} \mathrm{~s}$ there would be $\sim 10^{6}$ functional quanta sufficient to potentially affect chemical bonds. This would mean that for the $\sim 6 \cdot 10^{8}$ to $6 \cdot 10^{5}$ molecules that comprise these molarities, respectively, critical values could occur within one day to several days. This duration would be within the typical halflife of this class of proteins.

\section{BIOLOGICAL IMPLICATIONS FOR ADAPTATION OF THE SPECIES}

Assuming a more or less average value for current density of the earth's surface, magnetic permeability, and length of the average cerebrum the rate limiting step for the range of operating intensity for the transcerebral magnetic field (the energy induced within each cerebrum and the resulting wavelength for photons) would be determined by the total numbers of brains. With $\sim 7$ billion brains within the current population, the predicted magnetic field of $\sim 97 \mathrm{nT}$ would be associated with a wavelength of $405 \mathrm{~nm}$. Obviously this would not be an exact value but a centroid around which there would be normal distributions of intensities and hence corresponding wavelengths. Dependence of the effect upon the numbers of human cerebra appropriately "entangled" suggests there could be a critical mass of brains where qualitatively different phenomena appear.

The occurrence of the decreasing wavelength as a function of increasing numbers of brains within the homo sapiens sapiens, could contribute to the intrinsic structure of DNA within the species. Kielbassa et al. [42] found that induced DNA damage, as inferred from ratios of base modifications, from exposure to ultraviolet light was primarily between 290 and $315 \mathrm{~nm}$. There was a second maximum between 400 and $450 \mathrm{~nm}$. Recently we [43] found that exposure in the dark (photon flux density of $\sim 10^{-11} \mathrm{~W} \cdot \mathrm{m}^{-}$ ${ }^{2}$ ) of spring water to temporally patterned magnetic fields with intensities in the $0.1 \mu \mathrm{T}$ range produced shifts in peak wavelength, as measured by fluorescent spectroscopy, from $\sim 399 \mathrm{~nm}$ to $\sim 409 \mathrm{~nm}$. This $10 \mathrm{~nm}$ displacement of wavelength, which is the approximate width of a plasma cell membrane, was equivalent to $\sim 10^{-20} \mathrm{~J}$ shift in energy at this range in wavelengths. If we assume the coefficients for the powers of 10 employed for calculations in this paper are proximal to real values, then the probability increases that every brain of the species will be prone to similar and increasing modifications as the population increases. Although the energy would be very small and likely indiscernible within the temporal frames of human perception its effect would accumulate through the approximately 2 Gigaseconds of each person's life time.

The second implication of this approach, if valid, involves the emerging global importance of dreaming or REM (rapid eye movement) stages during sleep. The cerebral cortical activity during REM sleep is remarkably similar to the waking state except that neural input shifts dominance from thalamic to mesiobasal (hippocampal) sources of modulations and there is a statistical anisotropy from left to right hemispheric dominance. The durations of these periods, which occur about 4 to 5 times per night once every $90 \mathrm{~min}$ to $120 \mathrm{~min}$ in the majority of human beings, changes from about $10 \mathrm{~min}$ to $20 \mathrm{~min}$ as a function of the duration of the sleep cycle. The intrinsic integration between the mesiobasal temporal lobe structures and the cerebral cortices, an important component for cognitive processing, is created by the cross-frequency coupling of theta and gamma activity [44] between structures. Coherent $40-\mathrm{Hz}$ (gamma activity) oscillations characterize the dream state in humans [45].

The range of single REM episodes (10 to $20 \mathrm{~min}$ ) may be important because the estimated time for connectivity based upon magnetic diffusivity is the same duration. This sets the condition for emergent phenomena within the hundreds of millions of brains engaged in the same cerebral state at the same time over large expanses on the nocturnal side of the planet. Theoretically, the information from one brain could access all brains immersed within the medium within a single REM period. During REM periods there is a marked increase in protein synthesis associated with the consolidation of memories and the activation of geneticallydetermined mechanisms that control this process. That periods of quiet geomagnetic activity, when the effects of the steady-state field would be prominent, are associated with specific types of dream content described by the experients as "more bizarre" has been reported by Lipnicki [46]. 
The right hemisphere becomes relatively more prominent during dream sleep [47] and there are multiple experimental and correlational studies that strongly suggest the right hemisphere is particularly sensitive to normal geomagnetic intensities and their subtle fluctuations during perturbations within the magnetopause from changes in solar activity [48, 49]. One of the basic principles of Biology is that structure dictates function. If the sequences that contribute to the bases of memory produce coherence between a substantial subpopulation of the billions of brains within their dream states, then the details that are reconstructed as well as the generalized responses to stimuli would be affected during the following days.

\section{CONCLUSION}

The immersion of billions of human brains within a shared medium, the geomagnetic field, has the potential to influence the subtle physical-chemical changes within the entire species. The putative shift in secondarily induced energies from this shared immersion is associated with electromagnetic wavelengths that may soon reach a threshold to affect genetic expression. The threshold would be influenced primarily by the total number of cerebrums and may be occurring during the present biological interval involving the transition between 6 and 8 billions of brains.

\section{CONFLICT OF INTEREST}

The authors confirm that this article content has no conflicts of interest.

\section{ACKNOWLEDGMENTS}

The author thanks Professor Ghislaine F. Lafreniere for her technical support and for our many hours of thoughtful and enjoyable conversation.

\section{REFERENCES}

[1] Matsushita S, Campbell WH. Physics of geomagnetic phenomena. New York: Academic Press 1967.

[2] Saroka KS, Persinger MA. Potential production of Huglings Jackson's "parasitic consciousness" by physiologically-patterned weak transcerebral magnetic fields: QEEG and source localization. Epilep Behav 2013; 28: 395-407.

[3] Persinger MA, Richards PM. Vestibular experiences of humans during brief periods of partial sensory deprivation are enhanced when daily geomagnetic activity exceeds 15-20 nT. Neurosci Lett 1995; 194: 69-72.

[4] Mulligan BP, Persinger MA. Experimental simulation of the effects of sudden increases in geomagnetic activity upon quantitative measures of human brain activity: validation of correlational studies. Neurosci Lett 2012; 516: 24-56.

[5] Mulligan BP, Hunter MD, Persinger MA. Effects of geomagnetic activity and atmospheric power variations on quantitative measures of brain activity: replication of the Azerbaijani studies. Adv Space Res 2010; 45: 940-8.

[6] Babayev ES, Allahveriyeva AA. Effects of geomagnetic activity variations on the physiological and psychological state of functionally healthy humans: some results of Azerbaijani studies. Adv Space Res 2007; 40: 1941-51.

[7] Buckner RL, Petersen SE. What does neuroimaging tell us about the role of the prefrontal cortex in memory retrieval? Semin Neurosci 1996; 8: 47-55.

[8] Persinger MA. The effects of transient or intense geomagnetic or related global perturbations upon human group behavior. In: Calhoun JB Ed. Perspectives on adaptation, environment and population. New York: Praeger 1983; pp. 28-30.
[9] Blinkov SM, Glezer II. The human brain in figures and tables: a quantitative handbook. N.Y.: Plenum Press 1968.

[10] Winch DE, Ivers DJ, Turner JPR, Stenning RJ. Geomagnetism and Schmidt quasi-normalization. Geophys J Int 2005; 160: 487-504.

[11] Volland H. Quasi-electrostatic fields within the atmosphere. In Volland H (ed) Handbook of atmosphere Vol 1. Boca Raton (Fla): CRC Press 1982; pp. 65-106.

[12] Israel H. Atmospheric electricity: Volume 2 Fields, charges, current. Washington D.C.: Israel Program for Scientific Translations, 1973; p. 485.

[13] Koenig HL, Krueger AP, Lang S, Soenning W. Biologic effects of environmental electromagnetism. Berlin: Springer-Verlag 1981.

[14] Cho M, Rycroft MJ. Computer simulation of electric field structure and optical emission from cloud top to ionosphere. J Atmos Sol Ter Phys 1998; 60: 871-88.

[15] Halliday D, Resnick R. Physics fore students of science and engineering (combined edition). N.Y.: John Wiley \& Sons 1962.

[16] Persinger MA. Solutions for real values in Minkowski fourdimensional space may link micro-quantum and macro-quantum processes in the brain. Neurosci Biobehav Rev 2012; 36: 2334-8.

[17] Wikswo JP, Barach JP, Freeman JA. Magnetic field of a nerve impulse: first measurements. Science 1980; 208: 53-5.

[18] van Essen DC, Drury HA. Structural and functional analyses of human cerebral cortex using a surface-based atlas. J Neurosci 1997; 17: 7079-102.

[19] Persinger MA, Lavallee CF. Theoretical and experimental evidence of macroscopic entanglement between human brain activity and photon emissions; implications for quantum consciousness and future applications. J Cons Explor Res 2010; 1: 785-807.

[20] Persinger MA, Lavallee CF. The sum of $\mathrm{N}=\mathrm{N}$ and the quantitative support for the cerebral holographic and electromagnetic configuration of consciousness. J Cons Stud 2012; 19: 128-53.

[21] Di Biase F. A holoinformational model of consciousness. Quant Biosys 2009; 3: 207-16.

[22] Persinger MA. $10^{-20}$ Joules as a neuromolecular quantum in medicinal chemistry: an alternative to the myriad molecular pathways? Curr Med Chem 2010; 17: 3094-98.

[23] Persinger MA. On the possible representation of the electromagnetic equivalents of all human memory within the earth's magnetic field: implications for theoretical biology. Theor Biol Insights 2008; 1: 3-11.

[24] Bordag M, Mohideen U, Mostepanenko VM. New developments in the Casimir effect. Phys Rep 2001; 353: 1-205.

[25] Ryskin G. Secular variation of the earth's magnetic field: induced by ocean flow? New J Phys 2009; 11: 063015.

[26] Dotta BT, Persinger MA. "Doubling" of local photon emissions when two simultaneous, spatially separated, chemiluminescent reactions share the same magnetic field configurations. J Biophys Chem 2012; 3: 72-80.

[27] Kiwamu N, Kobayashi N, Fukao Y. Resonant oscillations between the solid earth and the atmosphere. Science 2000; 287: 2244-6.

[28] Persinger MA. Annual variation of local photon emissions' spectral power within the $\mathrm{mHz}$ range overlaps with seismic-atmospheric acoustic oscillations. Int J Geosci 2012; 3: 192-4.

[29] Li C-y, Poo M-m, Dan Y. Burst spiking of a single cortical neuron modifies global brain state. Science 2009; 324: 643-5.

[30] Cherry N. Schumann resonances, a plausible biophysical mechanism for the human health effects of solar/geomagnetic activity. Natural Hazards 2002; 26: 279-331.

[31] Hill RD. Spherical capacitor hypothesis of the earth's static electric field. Pure App Geophys 1971; 84: 67-74.

[32] Nikonenko I, Jourdain P, Alberi S, Toni N, Muller D. Activityinduced changes in spine morphology. Hippocampus 2002; 12: 585-91.

[33] Bokkon I, Dreams and neuroholography: an interdisciplinary interpretation of development of the homeotherm state in evolution. Sleep Hypnosis 2005; 7: 61-76.

[34] Dotta BT, Saroka KS, Persinger MA. Increased photon emission from the head while imagining light in the dark is correlated with changes in electroencephalographic power: support for Bokkon's biophoton hypothesis. Neurosci Lett 2012; 513: 141-54.

[35] Persinger MA, Saroka KS, Koren SA, St-Pierre LS. The electromagnetic induction of mystical and altered states within the laboratory. J Cons Explor Res 2010; 1: 808-30.

[36] Tsang EW, Koren SA, Persinger, MA. Power increases within the gamma range over the frontal and occipital regions during acute 
exposures to cererebrally counterclockwise rotating magnetic fields within specific derivatives of change. Int J Neurosci 2004; 114 : 1183-93.

[37] Lehmann D, Grass P, Meier, B. Spontaneous conscious covert cognition states and brain electric spectral states in canonical correlations. Int J Psychophysiol 1995; 19: 41-52.

[38] Gloor P. The temporal lobe and limbic system. Oxford: Oxford Press 1997.

[39] Angel A, Klink R. Differential responsiveness of stellate and pyramidal-like cells of the medial entorhinal cortex layer II. J. Neurophysiol 1993; 70: 128-43.

[40] Schlegel K, Fullekrug M. Schumann resonance parameter changes during high energy particle precipitation. J Geophys Res 1999; 104: 10, 111-10, 118 .

[41] Alberts B, Johnson A, Lewis J, Raff M, Roberts K, Walter P. Molecular biology of the cell. N.Y.: Garland Science, $2002\left(4^{\text {th }}\right.$ ed).

[42] Kielbassa C, Roza L, Epe B. Wavelength dependence of oxidative DNA damage induced by UV and visible light. Carcin 1997; 18: 811-6.

[43] Murugan NJ, Karbowski LM, Lafrenie, R, Persinger MA. Maintained exposure to weak $(\sim 1 \mu \mathrm{T})$ temporally patterned magnetic fields shift photon fluorescent spectroscopy in spring water but not ultrapure water: effects of different shielding materials, in submission.

[44] Whitman JC, Ward LM, Woodward TS. Patterns of cortical oscillations organize neural activity into whole-brain functional networks evident in the fMRI BOLD signal. Front Hum Neurosci 2013; 7: (article 80): 1-4.

[45] Llinas R, Ribary U. Coherent 40-Hz oscillation characterizes dream state in humans. Proc Nat Acad Sci USA, 1993; 90: 2078-81.

[46] Lipnicki DM. An association between geomagnetic activity and dream bizarreness. Med Hypoth 2009; 73: 115-7.

[47] Gordon HW, Frooman B, Lavie P. Shift in cognitive asymmetries between waking from REM and NREM sleep. Neuropsychol 1982 20: 99-103.

[48] Sandyk R. Improvement of right hemispheric functions in a child with Gilles de la Tourette's syndrome by weak electromagnetic fields. Int J Neurosci 1995; 81: 199-213.

[49] Belisheva NK, Popov AN, Petukhova NV, et al. Quantitative and qualitative evaluations of the effect of geomagnetic field variations on the functional state of the human brain. Biophysics 1995; 40: 1007-14.

(C) Michael A. Persinger; Licensee Bentham Open.

This is an open access article licensed under the terms of the Creative Commons Attribution Non-Commercial License (http://creativecommons.org/licenses/by$\mathrm{nc} / 3.0 /$ ), which permits unrestricted, non-commercial use, distribution and reproduction in any medium, provided the work is properly cited. 